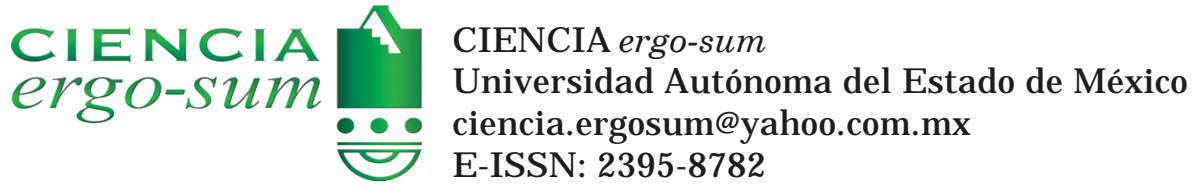

\title{
Propuestas técnico-administrativas para el sistema de producción de maíz. Estudio de caso: Rancho La Ciénega
}

González Pérez, Cándido; Uriega Chirino, Martha Alejandra

Propuestas técnico-administrativas para el sistema de producción de maíz. Estudio de caso: Rancho La Ciénega

CIENCIA ergo-sum, vol. 28, núm. 1, marzo-junio $2021 \mid$ e107

Universidad Autónoma del Estado de México, México

Esta obra está bajo una Licencia Creative Commons Atribución-NoComercial-SinDerivar 4.0 Internacional .

González Pérez, C. y U riega Chirino, M. A. (2021). Propuestas técnico-administrativas para el sistema de producción de maíz. Estudio de caso: Rancho La Ciénega . CIE NCIA ergo-sum, 28(1). https://doi.org/10.30878/ces.v28n1a1 


\title{
Propuestas técnico-administrativas para el sistema de producción de maíz. Estudio de caso: Rancho La Ciénega
}

\section{Technical-administrative proposals for the corn production system. Case study: Rancho La Ciénega}

\author{
Cándido González Pérez \\ Universidad de Guadalajara, México \\ cgonzalez@cualtos.udg.mx \\ (D) http://orcid.org/0000-0003-4876-5734 \\ Martha Alejandra Uriega Chirino \\ Universidad de Guadalajara, México \\ tepaale@hotmail.com \\ (D) http://orcid.org/0000-0002-6474-9948
}

Recepción: 04 de marzo de 2019

Aprobación: 27 de junio de 2019

\begin{abstract}
RESUMEN
Los resultados buscan demostrar que los esfuerzos individuales encaminados a la sustentabilidad de la producción agrícola pueden dar buenos resultados. Esta exposición corresponde a un estudio de caso del área de las ciencias sociales y está basado en la experiencia de dos productores del campo comprometidos con el respeto al medioambiente. Cuando la producción de alimentos se pone como principal objetivo la generación de ingresos, se atenta contra la naturaleza; aquí se muestra que puede haber compatibilidad entre ganancias y respeto ecológico cuando se aplican buenas prácticas de manejo de suelo y técnicas administrativas adecuadas.
\end{abstract}

PALABras CLAVE: productividad, rentabilidad, sustentabilidad, gestión.

\begin{abstract}
With the publication of this research results, we want to demonstrate that individual efforts that aim at the sustainability of agricultural production can give good results. This work corresponds to a case study in the social sciences, and is based on the experience of two farmers who work in the field and who are committed to respect the environment. When the food production is used looking for the generation of income as the main objective, it attacks nature. Here we demonstrate that there can be compatibility between profits and ecological respect when good soil management practices and adequate administrative techniques are applied.
\end{abstract}

KEYWORDS: productivity, profitability, sustainability, management.

\section{INTRODUCCIÓN}

El artículo se enfoca en tratar de identificar cuáles son los factores que influyen con mayor frecuencia en los parámetros del análisis de la rentabilidad en un rancho productor de maíz. Las características físicas del predio objeto de estudio indican que el tipo de suelo es arcilla, del tipo que tiene un grado alto de retención de agua y nutrientes con una conductividad hidráulica baja, lo cual representa una ventaja por tratarse de un cultivo de temporal; sin embargo, tiene la desventaja de que cuando las lluvias exceden el promedio puede dañar seriamente la cosecha. Según el INEGI, el suelo está clasificado como Planosol, que se refiere a zonas agrícolas dependientes del comportamiento de las lluvias. En aras de entender de qué manera se entrelazan los factores del sistema de producción (tierra, trabajo y capital), se decidió utilizar el instrumento de entrevista para que de una manera empírica las 
personas que han crecido en el campo aporten su experiencia. Importa saber cuáles son los principales elementos para saber hacia dónde se debe enfocar y poner todo el esfuerzo. Asimismo, interesa conocer cuál es la óptima utilización y aplicación de los componentes, es decir, identificar y aprovechar al máximo los conocimientos y las técnicas con las que cuentan las unidades de producción llamadas ranchos.

\section{Proceso preparación del suelo}

De forma preliminar, se ha procurado realizar la nivelación del terreno de forma muy limitada con el tractor y una cuchilla para tener un mejor drenaje del agua de lluvia. En este sentido, se ha usado un subsuelo para remover la tierra más dura y posteriormente se hace un manejo con rastra para dejarla más fina y tener mejor manejo, después se construyen zanjas para facilitar la salida del agua y finalmente se hacen los surcos para la siembra.

El predio se encuentra ubicado a 20 47'44.37” N y 102 25’08.76" O con una elevación de $2037 \mathrm{msnm}$. De acuerdo con el INEGI, el predio está dentro de una zona clasificada como Planosol, lo cual indica que son tierras altas húmedas o subhúmedas que se encuentran cubiertas por una capa de arcilla. Una medida aplicada desde que los nuevos propietarios hicieron uso del predio es sembrar desde las primeras lluvias para evitar problemas con el uso de los tractores porque el terreno se ubica en una ciénega.

\section{Compra de Semilla e insumos}

La semilla se compra en enero y se paga en efectivo para aprovechar los descuentos de preventa, y la entrega se efectúa a finales de abril. En general, el precio del maíz amarillo se ha considerado que maneja un precio más elevado que el maíz blanco, entre 10 a 50 centavos por kg, razón por la cual se sembró los primeros dos años dicha semilla; sin embargo, en ocasiones el precio se iguala. Posteriormente, se cambió a maíz blanco considerando la posibilidad de obtener una mayor producción por hectárea de acuerdo con la sugerencia del ingeniero agrónomo y proveedor; cabe mencionar que en esta etapa inicial se buscó la semilla que más se adaptara a las características del suelo.

El precio de los fertilizantes varía durante el temporal de siembra. Por lo general, se ha comprado cuando comienza la temporada para asegurar el abastecimiento y obtener un precio más bajo, ya que tiende a incrementar su demanda dentro del ciclo, y por ende el precio, además de estar sujeto a las fluctuaciones del dólar en el mercado, en específico el fertilizante DAP por ser de importación. La adquisición de otros fertilizantes como la urea y foliares, así como de los herbicidas e insecticidas necesarios, es relativamente fácil, pues se trata de una zona productora donde existe alta afluencia de insumos.

\section{Proceso de manejo del cultivo}

Al momento de la siembra, también se hace la primera aplicación del fertilizante llamado DAP (18-46-00) junto con un insecticida. Como su porcentaje de nitrógeno es bajo, es necesario complementar con urea para satisfacer las necesidades del suelo. La segunda aplicación se lleva a cabo a los 35 días de la siembra con urea, 250kg/ha, la cual se realiza con boleadora, y una tercera con urea, $250 \mathrm{~kg} / \mathrm{ha}$, a los 65 días con el uso de mochilas y de forma manual. Se siembra en seco y en las primeras lluvias se aplica un herbicida preemergente (atrazina). Una vez sembrado, en caso de presencia de hierba a los $20 \mathrm{~cm}$, se aplica un herbicida entre surcos.

\section{Proceso de desagüe}

El predio de estudio cuenta con una particular topografía, ya que dentro de él existe una gran acumulación de agua pues no se filtra con facilidad por lo que se han construido canales de desagüe debido a que se trata de una ciénega, es decir, un tipo de tierra que retiene mucha humedad. Debido a lo anterior, se han hecho excavaciones 
alrededor de las parcelas cuando es época de lluvias y así trasladar el agua hacia el exterior con la ayuda de una bomba eléctrica para evitar problemas de encharcamiento que afecten al cultivo

\section{Consumo nacional}

Es interesante abordar el tema del maíz por ser parte fundamental de la economía nacional y, por lo tanto, del producto interno bruto. En México es el principal cultivo dada su importancia en la ingesta alimenticia diaria de la población junto con el frijol. No obstante, de los más de 30 millones de toneladas que se consumen de forma interna, solamente 21.5 millones son producidas en el territorio. Es decir, hay déficit de $28 \%$ del consumo nacional (Núñez, 2016). Es crucial reconocer que el país tiene dificultades en la competencia de la producción respecto a otros (González y Alferes, 2010), además de las dificultades internas.

La Encuesta Nacional de 2014, en conjunto INEGI-Sagarpa, deja claro los profundos problemas en la producción agrícola interna, de la cual forma parte el maíz. Esta información muestra que más de la mitad de la superficie destinada a la agricultura es de agostadero, está enmontada o en descanso. Del total que realmente se siembra, $20 \%$ es de riego y $80 \%$ de temporal. Otra información interesante es el bajo nivel escolar de los productores, lo cual podría ser una de las causas por las que existe poca solicitud de créditos bancarios u otras fuentes de financiamiento. De los créditos otorgados, llama la atención que la mayor parte se destina a compra de semillas e insumos y pago de salarios y un porcentaje mínimo a la inversión en equipo de vanguardia e infraestructura. Esto ayudaría a explicar la baja implementación de innovaciones en el campo que, por un lado, limita el crecimiento de los negocios y, por otro, los flujos de efectivo se quedan cortos para hacer frente al capital de trabajo.

\section{6. ¿Por Qué estudio de Caso?}

Este artículo surge como una inquietud de los autores de desarrollar un negocio enfocado en la producción agrícola tomando en consideración que la siembra de maíz es uno de los granos más importantes del sector primario. Asimismo, la agricultura representa en muchos casos el patrimonio total para las familias dedicadas a este ramo. Es valiosa y novedosa la propuesta, pues se trata de presentar un trabajo con fundamentos metodológicos por medio de la implementación de elementos sistemáticos que sirvan de base para el conocimiento de un negocio propio. Se deben optimizar los recursos con los que se cuenta y las herramientas que la administración ofrece para el exitoso desarrollo del negocio de la agricultura. También se pretende ofrecer una visión más formal, ya que en Jalisco se ha empleado de manera empírica con poco control de la información sistemática. Existe un mercado nacional atractivo por cubrir y con ello promover la disminución paulatina de las importaciones de las cuales depende el país para abastecer sus necesidades.

\section{LA PRODUCCIÓN DEL MAÍZ COMO PARTE FUNDAMENTAL EN LA VIDA DE LOS MEXICANOS}

Teozintle, milli, cacabuacintle, totomoxtle y huitlacoche, entre otros vocablos para nombrar al maíz, tienen origen náhuatl. Su evidencia más antigua como alimento humano proviene de algunos lugares arqueológicos en México, lo cual reafirma el hecho de que éste y muchos de sus derivados son de origen mexicano. La tortilla de maíz es considerada patrimonio cultural de este país y es el eje de su cultura gastronómica al ser considerado el alimento por excelencia. Gracias a la tortilla puede externarse la identidad, así como desarrollar la imaginación y capacidad creativa en los más de trescientos platillos que de él derivan y se van dejando como legado de generación en generación, y que en la actualidad se manifiestan en diversas expresiones culturales que enorgullecen y distinguen a los mexicanos (Huerta, 2015).

El maíz es el cultivo gramíneo más importante en México con un 36\% de la superficie total cultivada. La producción ascendió a 25.4 millones de toneladas en el ciclo 2015-2016. La mayor parte de la producción es de 
maíz blanco (88\%); el resto es maíz amarillo (existe producción poco significativa de semillas y otras variedades). La productividad promedio en México es de aproximadamente 3.5 ton/ha. La mayor productividad promedio se asocia a la modalidad de riego en donde se alcanzan 8 ton/ha. En la modalidad de temporal, la productividad promedio es mucho más baja, pues se sitúa en 2.3 ton/ha.

México cuenta con infraestructura de riego nada más para $17 \%$ de la superficie sembrada, que se da sobre todo en el ciclo otoño-invierno y se concentra en Sinaloa. Esta entidad es la de mayor productividad promedio con alrededor de 9.7 ton/ha. Sinaloa, Jalisco, Michoacán, México y Guanajuato son las entidades con mayor rendimiento por hectárea y contribuyen con $56 \%$ de la producción.

La gráfica 1 indica la producción de maíz de grano en donde se aprecia que Jalisco cuenta con el segundo lugar después de Sinaloa según datos del Censo 2012 realizado por Sagarpa. Sin embargo, la mayor superficie sembrada corresponde a Chiapas, Veracruz, Oaxaca, Puebla y Estado de México que representan 40\%, pero tienen rendimientos menores. Lo anterior se muestra en la gráfica 2.

La gráfica 2 exhibe la participación de Jalisco en superficie sembrada de maíz grano con 605082 ha ubicándolo en segundo lugar a nivel nacional después de Chiapas, que cuenta con 705242 ha de superficie sembrada, según datos del Censo realizado en 2012 por Sagarpa. En términos generales, México es autosuficiente en maíz blanco, aunque en ocasiones ha tenido que importar este producto de Sudáfrica. El país posee deficitario en maíz amarillo, que se importa fundamentalmente de Estados Unidos. En 2016 las importaciones de maíz amarillo sumaron 2363 millones de dólares y representaron casi 13 millones de toneladas.

El consumo total de maíz en 2016 fue de casi 37 millones de toneladas de ambas variedades. La balanza comercial tuvo un déficit de 12.4 millones de toneladas, de tal forma que una tercera parte del consumo del grano fue atendido con importaciones (Núñez y Sempere, 2016). De este modo, en México es necesario que se evalúe su producción de maíz. Después de la liberación de aranceles y cuotas a las importaciones de ese grano, es importante mencionar que $43.2 \%$ de la producción total nacional tiene ventajas en la comparación con la producción internacional, la cual podría incrementarse si se implementara una política agrícola integral y eficiente (González y Alferes, 2010) dirigida a la intensificación sustentable de la agricultura (Leiva, 1998).

Desde 1973 se revisan hechos y experiencias de la administración aplicada al medio rural y aparece incluso como una disciplina académica en la educación superior. El país necesita de personas calificadas que resuelvan problemas administrativos en cualquiera de las actividades agrícola-industriales si se pretende salir del subdesarrollo donde el país se encuentra inmerso. Para estar en pos de la eficiencia y exigencia que impone la economía mundial, se deben optimizar los recursos. La administración de empresas juega un importante papel (Longenecker et al., 2012); sobre todo, la administración agropecuaria deberá ser un magnífico instrumento para alcanzar la anhelada autosuficiencia alimentaria (Aguilar y Cabral, 2000).

Dentro de los insumos sustanciales en agricultura, Luna et al. (2016) y Luna et al. (2012) coinciden en que la implementación de semilla mejorada es una gran medida de incremento a la productividad, ya que en la actualidad cuentan con diversas características que las hacen muy atractivas dependiendo de las características del suelo y del clima en donde se cultiven. La adopción de dichas semillas se ve favorecida por los programas públicos de agricultura por contrato, financiamiento e infraestructura para sistemas de riego para el desarrollo de la actividad.

Además, se debe incidir en los trabajos relativos al análisis de suelo, así como a todas las referidas al abono y a la eliminación de enfermedades y plagas, independientemente de las características de la zona donde se realice el cultivo. México es uno de los países con más diversificación de la producción agrícola del mundo. Existe al menos un tipo de sistema agrícola de cultivo de maíz característico de cada zona ecológica y también pueden identificarse sistemas de cultivo que van de los altamente intensivos en el uso de insumos hasta sistemas con muy bajo uso en tierras de temporal. Existe una enorme variedad de sistemas de cultivo maiceros y su estrecha relación con la riqueza ecológica y cultural del país. El cultivo de maíz es versátil y se ha desarrollado con pocas pausas, es decir, ha sido eficiente y continuo frente a los cambios naturales (Aguilar et al., 2003). 


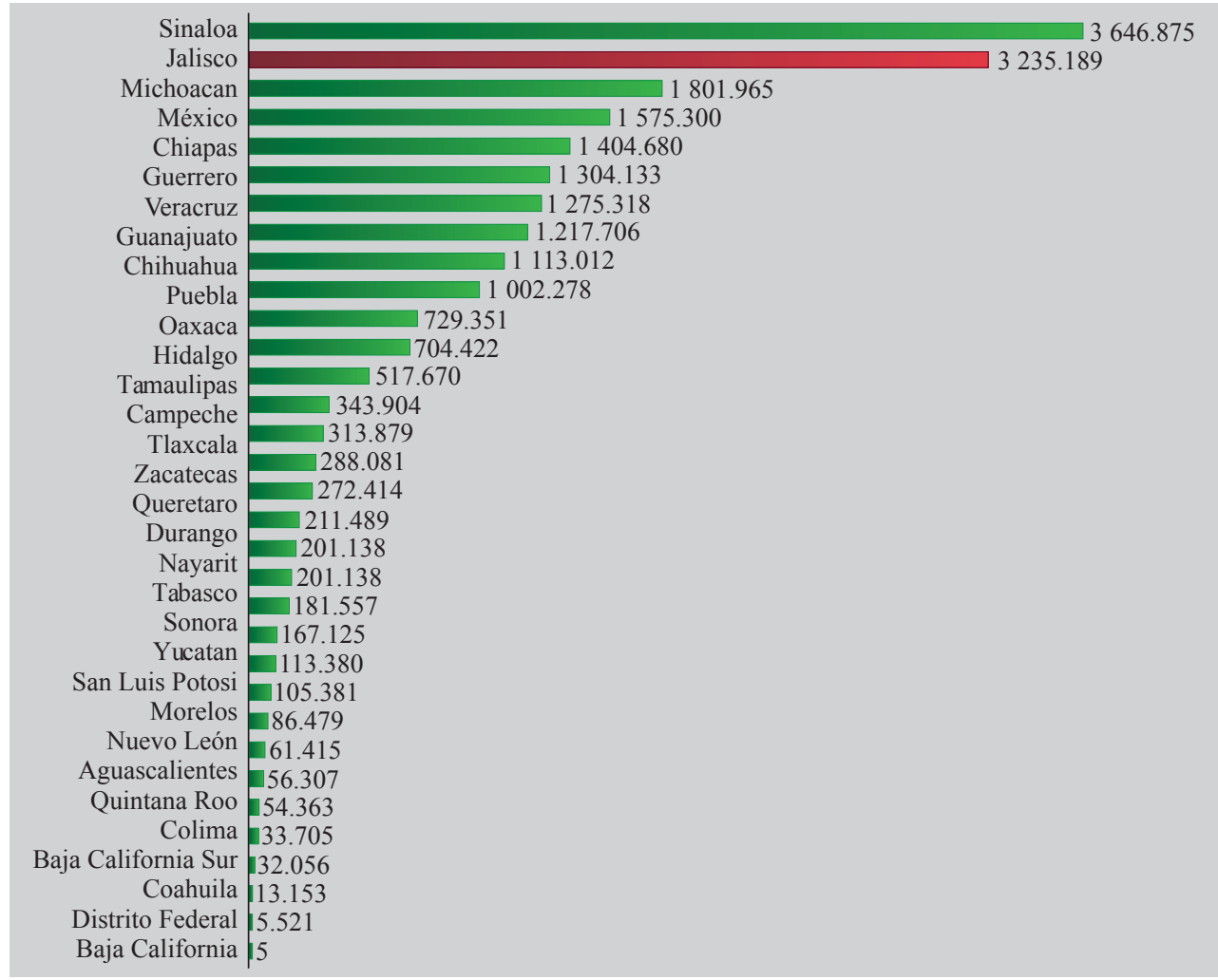

GRÁFICA 1

Participación de maíz grano, 2012 (toneladas)

Fuente: Sagarpa (2012).

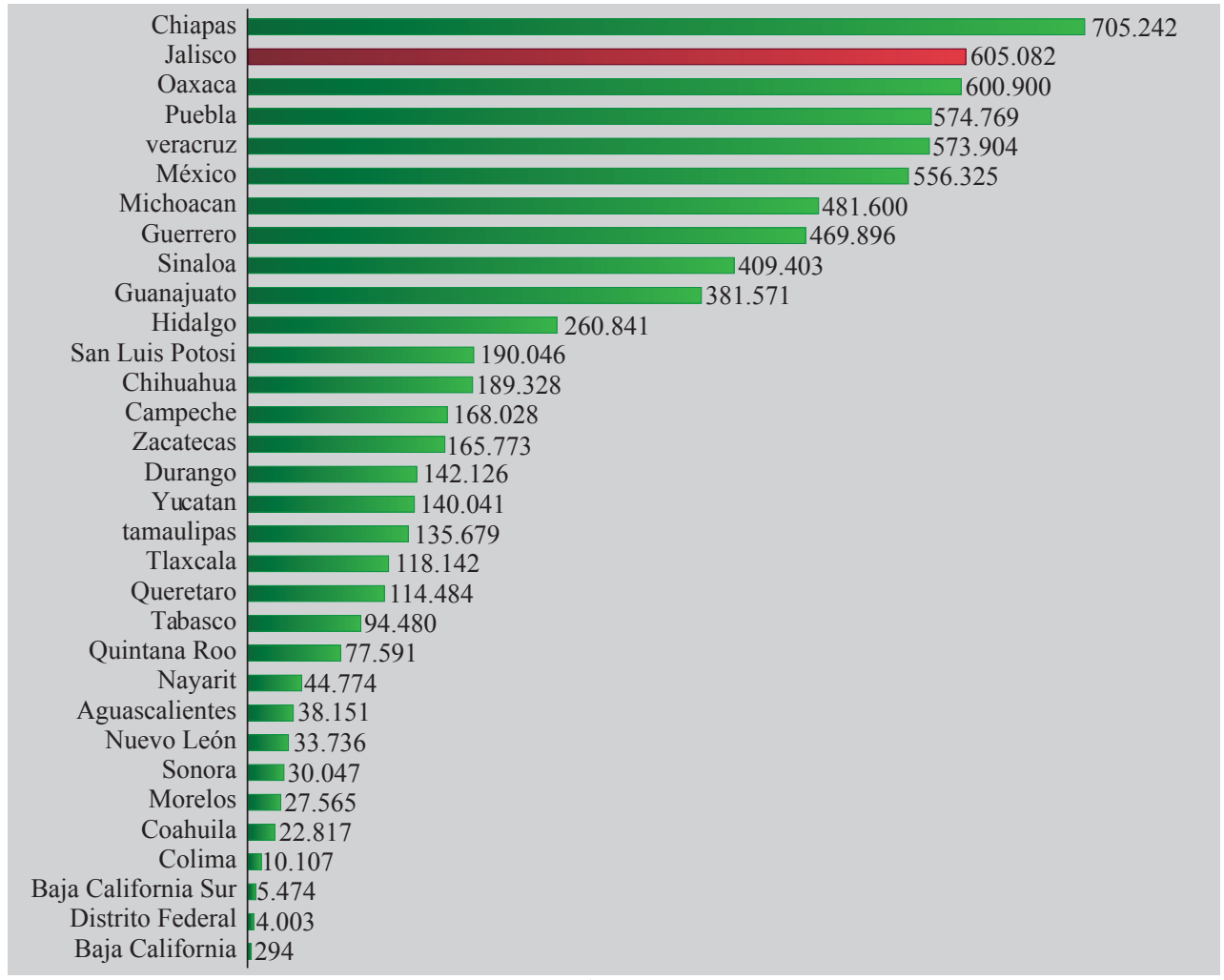

GRÁFICA 2

Participación de maíz grano, 2012 (hectáreas)

Fuente: Sagarpa (2012). 
La agricultura en nuestro país es una de las actividades de mayor relevancia en la economía, y la siembra de maíz es una de las más importantes tanto por la generación de ingresos como por la tradición alimentaria de nuestro país. Asimismo, es uno de los inventos más relevantes que ha realizado la humanidad, inclusive, con su aparición, los historiadores le han asignado un periodo especial que data de hace 10000 años aproximadamente (el Neolítico). Es, en resumen, la actividad económica por medio de la cual los hombres satisfacen sus necesidades de alimentación y de sobrevivencia (López, 2013). Sin embargo, los agricultores no siempre obtienen un rendimiento esperado en sus cultivos. Por ello, este artículo pretende evaluar si al mejorar la gestión administrativa y al hacer eficientes los recursos tecnológicos para el manejo de sus cultivos, se incrementa la rentabilidad (Aguilar y Cabral, 2000).

\section{La PRODUCCión de Maíz EN JALISCo}

Jalisco gracias a sus condiciones climáticas destaca en territorio nacional por su producción en maíz y también de semilla. Si bien esto representa una ventaja para los productores, también es una situación de conflicto, ya que producen con altos costos de insumos y tienen que competir con empresas transnacionales que han monopolizado el mercado. Jalisco se ubica en el segundo puesto en el liderazgo nacional de la producción de maíz de temporal, ya que en maíz de riego el productor más importante es Sinaloa.

En 2012 a nivel nacional una de cada tres hectáreas cultivables fue de maíz con un 91\% de blanco para consumo humano. Entre Sinaloa y Jalisco aportan un tercio de la producción nacional del cual es autosuficiente; sin embargo, importa cerca de 9.4 millones de toneladas de maíz amarillo para el sector pecuario (INEGI-Sagarpa, 2014).

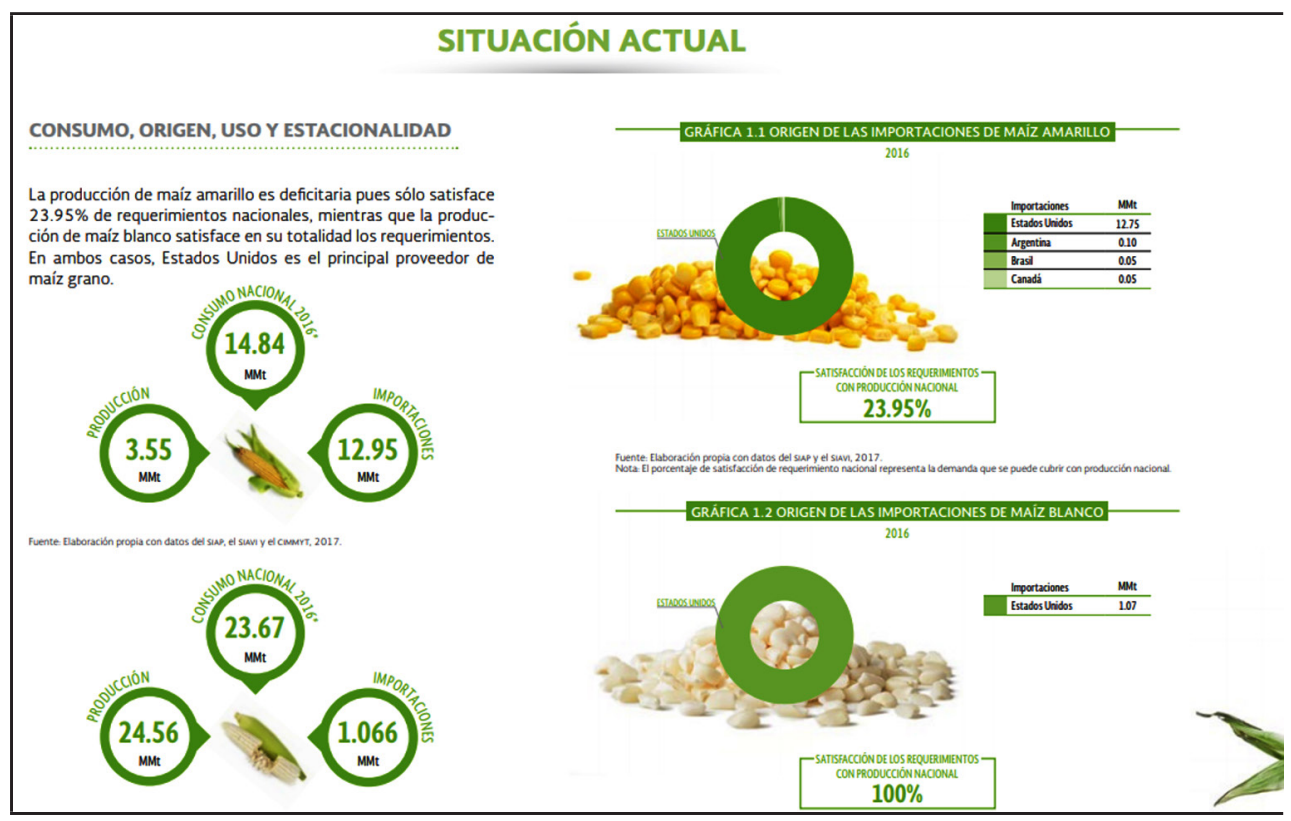

FIGURA 1

Situación actual

Fuente: Sagarpa (2017).

El maíz es la siembra más importante del estado, la cual se favorece cuando se tiene una buena cantidad de lluvia originada desde el Pacífico que riega la parte occidental del país (Castañeda et al., 2014). Específicamente, en la Región Altos Sur sobresalen los municipios Lagos de Moreno, La Barca y Tepatitlán de Morelos. Sin embargo, ha habido momentos de incertidumbre cuando las escasas lluvias de algunos temporales ponen en riesgo las expec- 
tativas de producción del grano en la entidad. La sequía es un problema que han enfrentado productores alteños durante varios años; no obstante, no es la única causa que determina el costo del grano. Arandas tiene 9938 ha de superficie cosechada con $1.70 \%$ de participación estatal, lugar en donde se encuentra el predio de estudio.

\section{LA RENTABiLIDAD}

La rentabilidad se puede definir en su forma más simple como una razón económica que compara los beneficios obtenidos en relación con recursos propios de la empresa. Es decir, obtener más ingresos que mermas en un campo determinado. Dicho de otra manera, es el rendimiento o ganancia que produce una empresa. Académicamente, se le denomina gestión rentable cuando un negocio no sólo evita las desventajas, sino que además permite obtener una ganancia a un plusvalor por encima del total de expendios de la empresa (Espinoza, 2011). Dentro de la teoría revisada en relación con la rentabilidad de cualquier negocio o inversor, se menciona que los activos o ganancias no necesariamente deben de tener un nivel bajo de peligro. En este caso, el papel del asesor financiero es buscar la manera de hacer crecer la rentabilidad; por supuesto, los riesgos no pueden disminuirse a $0 \%$; sin embargo, es el punto donde se debe poner la mayor atención.

La teoría de recursos y capacidades (Grant, 1997) explica que las empresas deben centrar su atención en identificar, desarrollar, proteger y desplegar aquellos recursos que son una ventaja competitiva sostenible y por lo tanto obtener rentas (rentabilidad). Preocupándose por los procesos dinámicos y el comportamiento organizativo, Grant (1997) trata conceptos fundamentales como competencias distintivas, rutinas organizativas, ventaja competitiva, rentabilidad corporativa y aprendizaje organizacional.

\section{TeOría de la Sustentabilidad}

En el ámbito académico es bien conocido el Informe Brundtland, que en 1987 se presentó ante la Comisión Mundial del Medio Ambiente y Desarrollo (creada en 1983) de la ONU; en el documento se establece la teoría del desarrollo sustentable que tiene como fin principal la protección de los recursos naturales, los cuales engloban los aspectos tanto de la vivienda y alimentación como del trabajo. Se hace hincapié en la satisfacción de esas necesidades fundamentales para las generaciones futuras. Por supuesto, el detrimento del entorno ambiental es la principal preocupación de este informe y hace una crítica de las formas de crecimiento económico que prevalecen en nuestro mundo, algunas desdeñan los daños que se hace a la naturaleza. Es imposible pensar que se debe dejar de producir para evitar daños al entorno, pero es imprescindible buscar nuevas formas de producción que sean amigables con lo que nos rodea. Por mucho tiempo no se consideró el deterioro del medioambiente; sin embargo, hoy se debe hacer un profundo análisis al tomar decisiones en donde estén presentes las creencias, valores y aspiraciones de esta nueva sociedad. Dentro de un paradigma tradicional de desarrollo de proyectos, se considera la vialidad y factibilidad al establecer primordialmente: evaluación-impactos y evaluación-sustentabilidad (González y Sánchez, s/f)

\section{EnTrevistas}

Se utilizó el método de entrevistas a profundidad a agricultores con experiencia en el manejo del cultivo de maíz, así como a algunas personas claves que aportaron información valiosa acerca de cómo manejar los aspectos contables y técnicos de la producción en la zona de La Ciénega de los Altos de Jalisco (Hernández-Sampieri, 2015); asimismo, se utilizó la técnica de historias de vida. Lo fundamental es describir ampliamente las experiencias que tienen los actores principales con respecto (en este caso de estudio) a la adquisición del predio y los planes de crecimiento que se tiene. Interesan los detalles personales, es decir, por qué se han hecho las cosas, cómo se ven dentro de un plazo mayor a cinco años, y todos los aspectos individuales que tengan relación con la experiencia y 
panorama que refleja la producción agrícola en el predio (Cordero, 2012). Los tópicos de las entrevistas oscilaron entre los aspectos del análisis del suelo, la nivelación, elección de semilla, el tipo de fertilizantes, los herbicidas, los insecticidas y los factores climatológicos.

\section{Buenas prácticas en el proceso de producción}

La primera de las acciones que se deben cumplir es la conocida coloquialmente como barbecho, la cual consiste en voltear la tierra por medio de un arado. Las actividades se deben llevar a cabo en la región donde se ubica el predio de esta investigación entre el 23 de enero y hasta el 5 de mayo (la fecha promedio es el 31 de marzo). El tipo de arado usado está compuesto por discos y consiste en voltear la tierra para nutrirla de nitrógeno; esto se logra porque se utiliza el rastrojo que ha quedado en el predio después de la cosecha anterior. Los desechos -el rastrojo citado - son muy beneficiosos para el mejoramiento de la tierra porque la hace más suave, más manejable y reintegra material orgánico para volver a producir. Este primer paso es decisivo para obtener mejores resultados en la cosecha y proviene del conocimiento popular compartido desde tiempos remotos en que se ha laborado la tierra para la reproducción del maíz.

Otro proceso que se ha ejecutado ancestralmente después del barbecho es el rastreo. Esta actividad la cumple 100\% de los campesinos de Los Altos de Jalisco. Para esto, se manipula una herramienta, la rastra (de ahí el nombre de rastreo), y consiste en pulverizar el terreno que va a sembrarse. Por la constitución de la tierra, cuando se seca se apelmaza y se forman los terrones y se pierde mucho en la calidad de la siembra cuando no se tiene el cuidado de desbaratarlos. El cultivo del maíz requiere en la medida de las posibilidades que la tierra se transforme en polvo; el rastreo se concreta entre el 8 de marzo y el 3 de junio (la fecha promedio es el 25 de mayo; en ocasiones se adelanta el periodo de lluvias y hay humedad suficiente para la germinación del grano). El proceso de rastreo hace posible incorporar mayor cantidad de rastrojo y se realiza después del barbecho, que provee sin duda una mayor cantidad de nitrógeno a la tierra.

Previo a la siembra, acontece otra actividad del conocimiento práctico de los campesinos: el acuartelado. Consiste en dividir la superficie destinada a la producción de maíz en cuadrículas por medio de zanjas con la finalidad de sacar el mayor provecho del agua de lluvia. Cuando las precipitaciones son copiosas, las zanjas sirven como desagüe y cuando son escasas mantienen más húmeda el área porque los surcos son menos profundos.

La última actividad es la siembra, la cual se lleva a cabo en seco porque se facilitan todas las actividades relacionadas con el depósito de la semilla. Cuando las lluvias son tempraneras, hay ocasiones en que se dificulta el ingreso del tractor, por eso es conveniente empezar en ese momento. Las fechas propicias son entre el 25 de junio y el 1 de julio. La totalidad de las personas entrevistadas como expertos en los temas de la siembra y cosecha de maíz coincide en que se debe utilizar semilla mejorada y de preferencia de semilla blanca, que se conoce como de "ciclo intermedio" porque tiene una duración de dos meses y medio en promedio. Se aplica una siembra de 90 000 simientes por hectárea. Las principales ventajas de utilizar esta semilla es que estas son tratadas para ser más resistentes a diversos aspectos del medioambiente cómo la humedad o la sequía; al respecto, hay de ciclo corto, medio y largo y con una serie de características que las hacen muy atractivas dependiendo del tipo de suelo que se pretende cultivar, pero por supuesto su costo también es mucho más elevado en función de las bondades que ofrece.

La fertilización más recomendada antes de empezar con los trabajos de la siembra es la aplicación de gallinaza. Se le denomina de esta manera al estiércol de gallina y ha sido aplicada de forma recurrente en toda la región porque es un "producto" que prolifera, puesto que la avicultura en Los Altos de Jalisco ocupa los primeros lugares de producción nacional. Los resultados han sido muy satisfactorios y son de origen orgánico. Esta práctica se ha repetido durante los últimos 50 años en la región y los campesinos la aplican variando un poco en su instrumentación; algunos hacen pequeños montículos en seco y por medio de una escrepa que le adaptan al tractor la van extendiendo de manera uniforme. Hay quienes rentan equipos a campesinos con mayores recursos, quienes cobran por prestar esos servicios. Otro tipo de fertilización, ya más industrial, consiste en aplicar la fórmula 18-46-00 
también con urea. Una se aplica a los 45 días de la siembra y la otra a los dos meses cuando la planta ya está muy desarrollada. La gallinaza mantiene la fertilización durante dos y hasta tres años; las otras son específicas del ciclo. Ya son muchos los campesinos que elaboran sus respectivos análisis de suelo y con eso la fórmula aplicada es más específica. No son pocos quienes están aplicando urea de lenta liberación y con eso pueden omitir la fórmula 18-46-00; los entrevistados afirman que obtuvieron resultados muy positivos.

La escarda es un proceso posterior a la siembra. Consiste en oxigenar el suelo y eliminar las hierbas que empiezan a proliferar alrededor de las plantas de maíz. Se utiliza un implemento que se adapta al tractor cuando la milpa está pequeña aún. Esta actividad en particular es de las menos aplicadas; sin embargo, como se han visto buenos resultados, ha ido al alza. Se ha observado que cuando se lleva a cabo la escarda, se limita el uso de herbicidas y eso es muy benéfico para la tierra toda vez que se evita sobrecargarla de sustancias químicas no orgánicas. Es una de las mejores medidas para preservar el medioambiente natural.

El control de malezas y plagas de suelo es un procedimiento que se aplica por medio de insumos químicos; aunque es de los menos recomendados, es necesario hacerlos porque en ocasiones no hay otra opción. Se hace generalmente entre los quince días y las tres semanas después de la escarda. A últimas fechas, esta actividad se realizado a través de la renta de servicios de avioneta, y se omite solamente cuando las condiciones del terreno, o por lo pequeño de los predios, no lo permiten. La ventaja de esto queda a la vista: se elimina gran cantidad de trabajo manual y los costos disminuyen porque se combina la aplicación de herbicidas, fungicidas e insecticidas. Un beneficio extra lo constituye el hecho de que se evitan enfermedades y accidentes en los trabajadores puesto que el manejo es peligroso. Una práctica recurrente es que a los 45 días de la siembra se procede a la aplicación de urea, como ya se mencionó; la de lenta liberación ha proliferado en su uso porque tiene un rango mucho más alto de efectividad.

Para el control de maleza, cuando se hace necesario, se utilizan productos químicos, pero lo mejor es tratar de evitarlo en la medida de lo posible con las escardas. Los primeros 45 días son fundamentales para esta actividad porque se busca eliminar la competencia del crecimiento de la planta de maíz con otras por su lucha por la luz, el agua y los nutrimentos. La aplicación de foliares es de las últimas actividades y su objetivo principal es para el crecimiento del grano; ésta se lleva a cabo gracias a una avioneta cuando las condiciones lo permiten.

La trilla se aplica cuando las mazorcas presentan un 14\% de humedad. Este procedimiento es la última etapa de la siembra y cosecha del maíz. La administración financiera de la siembra y cosecha del maíz es una de las etapas fundamentales en esta investigación, por lo que forma cuerpo aparte de los procedimientos directos del trabajo en el campo. Las entrevistas en forma lírica dirigidas a los campesinos que han labrado sus tierras y han aprovechado los conocimientos de sus antepasados se extienden a la administración de la producción; por esa razón, se va a explicar en forma detallada los aportes que han expresado en forma oral. Es un hecho muy conocido en la región que ellos, los campesinos, han desarrollado la agricultura a pequeña escala y han contribuido con su grano de arena al desarrollado del país. El trabajo del campo, llevado a cabo generalmente por personas de bajo o nulo grado escolar, ha sido el sostén de millones de personas en México; por esta razón, no podemos excluir los aportes que los campesinos ofrecen en el ámbito administrativo. Es de suma importancia no olvidar que, pese a todos los trabajos y los esfuerzos por llevar controles más estrictos en todos los sentidos, el elemento decisivo es el temporal; sin embargo, si a un buen temporal aunamos que se han cuidado todas las demás variables podemos considerar que la cosecha puede ser altamente beneficiosa.

\section{Resultados de entrevistas}

Los cinco entrevistados coinciden en que se debe analizar el suelo. Uno de ellos indica que con realizarse ocasionalmente es suficiente, ya que él cuenta con experiencia para saber qué nutrientes le falta a su predio. Dos más están de acuerdo en que debe ser con cierta regularidad, aunque no sea necesariamente cada año, para conocer la situación que presenta y tener más confianza en los fertilizantes que se tendrían que aplicar. Los 
otros dos consideran que tendría que hacerse cada año para que se determine cuáles son las necesidades exactas de nutrimentos que requiere la tierra y estar en posibilidad de cubrirlas con la aplicación de los fertilizantes correspondientes.

En cuanto a la nivelación del suelo, los cinco entrevistados consideran que es indispensable en el área de La Ciénega para evitar que se creen zanjones y para una mejor distribución del agua. Cuatro de ellos comentan que hacerlo mecánicamente da buenos resultados, pues el uso del tractor agiliza el proceso y abarata los costos. Uno de ellos indica que si se realiza un año sí y otro no, teniendo cuidado con el proceso de rastreo y construyendo unas zanjas dentro del barbecho para transportar el agua fuera de la zona de cultivo, es suficiente. Uno más indica que cada dos o tres años está bien y dos de ellos coinciden en hacerlo cada año; sin embargo, el último de los entrevistados considera importante realizar este proceso vía satélite que si bien resulta costoso, ya que se tiene que contratar el servicio con equipo especializado, los beneficios son realmente considerables puesto que se obtiene una distribución de la humedad mucho más uniforme y controlada por mucho tiempo.

Referente al uso de semilla mejorada, los cinco entrevistados coinciden en que es indispensable su empleo conforme al temporal dentro de cada región. Generalmente, en esta zona se utiliza el maíz ciclo medio, ya que el temporal no suele ser tan largo, es decir, dura aproximadamente del 15 de mayo al 30 de octubre. En La Ciénega se mantiene por más tiempo la humedad, situación que ayuda en caso de que el temporal se retire pronto. Todos coinciden en que a pesar de ser muy cara la semilla mejorada, tiene buen rendimiento en la producción del grano y del rastrojo. En definitiva, ninguno se va por la siembra de maíz criollo, pues sus cañas son demasiado altas, las raíces no se anclan con firmeza al suelo y es muy fácil que se puedan caer con vientos o granizadas y mermar la producción.

Tres de los entrevistados consideran que nunca debe faltar la fórmula DAP 18-46-00 al momento de la siembra, ya que por el alto contenido de nitrógeno que contiene este abono es indispensable para la nutrición de la semilla. Uno de ellos prefiere aplicar una mezcla que hacen en las tiendas de agroquímicos por ser más económica que el DAP. Otro considera que elaborar ellos mismos la mezcla de acuerdo con las necesidades que reporta el análisis de su suelo les ha resultado muy beneficioso.

A grandes rasgos, es visible que la tendencia es aplicar el abono de gallinaza debido a que es muy notorio el beneficio de su aplicación. Cuatro de los entrevistados están de acuerdo en aplicarlo cada año y uno considera que cada dos o tres años es suficiente porque perdura su beneficio durante este tiempo. Sin embargo, no siempre la pueden aplicar, ya que se incrementan los costos considerablemente.

Tres entrevistados coinciden en aplicar urea en dos etapas, incluso uno de ellos recomienda la que es de lenta liberación. Se efectúan dos aplicaciones de urea: una a los 45 días de la siembra, tiempo que dura el efecto de la fórmula DAP, y otra a los dos meses de la siembra. Recomiendan foliares al momento que va a espigar la caña, lo cual sirve para que los granos de las orillas de las mazorcas se llenen al final del temporal, siempre y cuando los presupuestos lo permitan. Dentro de los fertilizantes naturales empleados, se encuentra la integración del rastrojo, que permite obtener una mayor nutrición y a su vez una consistencia más suave al suelo y propicia un manejo más fácil evitando que se formen duros terrones.

Todos los entrevistados coinciden en que la aplicación de los herbicidas, funguicidas e insecticidas es indispensable en el proceso de la siembra del maíz, ya que de lo contrario representa una merma de aproximadamente $50 \%$, al competir diversas plagas con el cultivo. Se considera su aplicación con avioneta, pero eso depende del lugar del predio, además de considerarse el incremento en los costos y el daño al medioambiente

Los factores climatológicos no son determinantes en esta zona del país. Esporádicamente, pueden presentarse granizadas, heladas o fuertes vientos que no afectan de manera importante; lo más delicado sería la falta de lluvia, sobre todo al final del ciclo. Tal vez, las lluvias que se desplazan hacia esta zona han beneficiado el cultivo. Las cinco personas a quienes se les realizó la entrevista presentan coincidencias en cuanto a que el factor más importante de todo el ciclo de producción es un buen temporal, aunque en el último de los casos se tengan que realizar labores de desagüe; uno de ellos manifiesta no escatimar en los insumos siempre que el presupuesto lo permita. 
Referente a la administración, cuatro de los entrevistados comentan que no es importante contratar a un contador debido a que siempre han vendido su producción sin que les exijan factura, siendo su pago en efectivo. Comentan que ellos mismos llevan las cuentas de sus gastos y de sus ingresos. En contraste, el último entrevistado refiere como esencial el implemento de un proceso contable para estar en posición de poseer elementos que permitan una acertada toma de decisiones; sin embargo, coincide en tener un apego puntual a cada una de las acciones que se involucran en el trabajo de la siembra sin escatimar en insumos ni en procesos.

\section{Conclusiones}

Se concluye que las buenas prácticas adoptadas en la producción de maíz, así como la integración de un programa administrativo que permita identificar los costos detalladamente, influyen positivamente en la productividad y, por lo tanto, en la rentabilidad del rancho agrícola (cuadro 1). En el cuadro 1 se detallan los ingresos y egresos por año desde que se inició la actividad de siembra en el rancho objeto de estudio. Como se puede apreciar, ha habido una proporción moderada de ingresos contra gastos de alrededor de $50 \%$ en los primeros cuatro años. Sin embargo, para la cosecha de $2018^{*}$ se obtuvieron ingresos por sólo $\$ 312850.00$ pesos mexicanos aun sembrando todo el terreno, es decir, 30 ha, ya que se tuvo que resembrar y se incrementaron los gastos, aunado a que el temporal fue corto; esto ocasionó que no se terminaran de llenar las mazorcas y que no se cumpliera con el peso específico, lo cual provocó pérdidas en ese año por 21020 pesos mexicanos (gráfica 3).

\section{CUADRO 1}

Inversión por hectáreas

\begin{tabular}{|lcccccc|}
\hline & Ingresos & Egresos & Kilogramos & Hectáreas & Precio unitario & $\%$ egresos/ingresos \\
\hline 2014 & $\$ 229274.00$ & $\$ 79060.00$ & 79060 & 15 & 2.90 & 34.48 \\
2015 & $\$ 321865.00$ & $\$ 175000.00$ & 56260 & 15 & 2.95 & 54.37 \\
2016 & $\$ 315186.55$ & $\$ 183370.00$ & 87590 & 20 & 3.60 & 58.18 \\
2017 & $\$ 547923.00$ & $\$ 291717.34$ & 140630 & 15 & 3.85 & 53.24 \\
$2018 *$ & $\$ 312850.00$ & $\$ 333910.00$ & 86340 & 30 & 3.60 & 106.73 \\
2019 & $\$ 686364.00$ & $\$ 271082.00$ & 163420 & 20 & 4.20 & 39.50 \\
\hline
\end{tabular}

Fuente: elaboración propia.

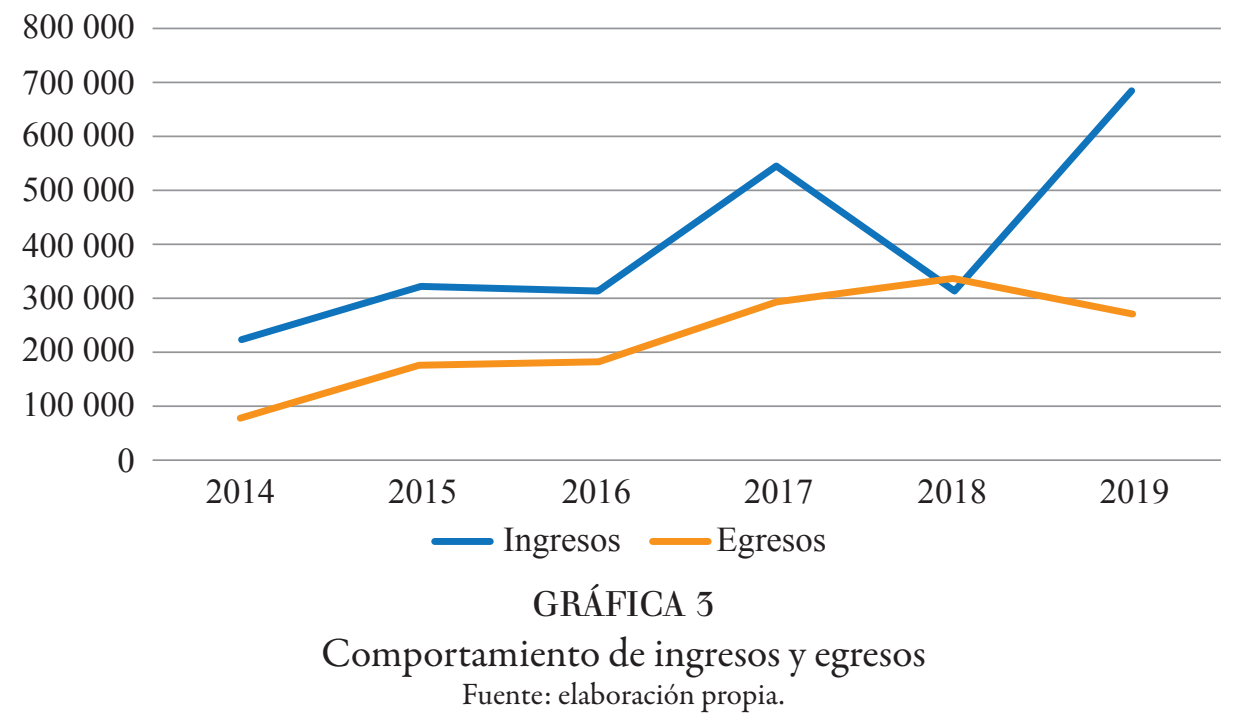


Como se puede observar en la gráfica 3, para la cosecha de enero de 2019 se lograron reducir los costos a 39.50\% debido al buen temporal de lluvias registrado durante 2018 y al apego de las buenas prácticas en el proceso de producción detallado en este trabajo. Además, se ha tenido especial cuidado en implementar de manera paulatina los siguientes procesos: análisis del suelo al principio de cada año, lo que ha permitido tener más certeza de los nutrientes que se deben aplicar; también durante el ejercicio 2017 se realizó la nivelación del suelo vía satélite que, aunque es un proceso costoso, ya que se tiene que contratar servicios profesionales, se apreció una distribución mucho más uniforme de la humedad directamente en el aumento de la producción en zonas que presentaban problemas de encharcamiento. La aplicación de gallinaza ha significado una muy buena fuente de nutrientes que también resulta ser más costosa que los abonos químicos, pero tiene resultados por más tiempo al evitar una aplicación adicional cuando se utilizan sólo abonos químicos, como es en el caso de la urea donde forzosamente se tienen que hacer dos aplicaciones durante el periodo de cultivo. Otro de los aspectos de mayor importancia es la utilización de semilla mejorada, también costosa. No obstante, cada vez se ofertan más semillas que se adaptan mejor para cada tipo de suelo, presentan mayor resistencia a ciertas plagas y con mayor rendimiento, incluso algunas ya cuentan con un insecticida incluido que evita la aplicación adicional de esta sustancia al momento de la siembra y permite un moderado ahorro; aun así, es recomendable estar en constante observación del cultivo por posibles plagas o comportamientos de la siembra y tomar decisiones oportunas de eventualidades que se puedan presentar, como es el caso de la plaga maicillo que se ha presentado en este rancho y que ha repercutido en costos de tiempo y dinero, pero gracias a los trabajos de manejo de la tierra se ha podido eliminar paulatinamente. Respecto a la parte administrativa, es muy importante considerar aspectos que comprenden hacer una buena selección de proveedores y prestadores de servicios para obtener insumos y servicios de calidad a precios razonables e identificar clientes cercanos a la zona, que evitarían riesgos por transporte e incremento en costos por fletes; además, llevar un control puntual de los costos ha permitido contar con información para tomar decisiones importantes en la implementación de medidas más adecuadas, por lo que se considera adecuado apegarse al siguiente paquete tecnológico por hectárea para áreas de temporal propuesto por una empresa distribuidora y productora de agroinsumos del municipio de Tlajomulco de Zúñiga (tabla 1) para manejo del cultivo en la medida que los tiempos y los factores meteorológicos lo permitan y así evitar salirse del presupuesto, pero sin arriesgar la calidad y cantidad de los insumos que repercutiría directamente en la rentabilidad de la producción. En este sentido, y de acuerdo con la tendencia de producción por hectárea que muestra la gráfica 4, se considera poder alcanzar una producción de 11 ton/ha en dos o tres años más.

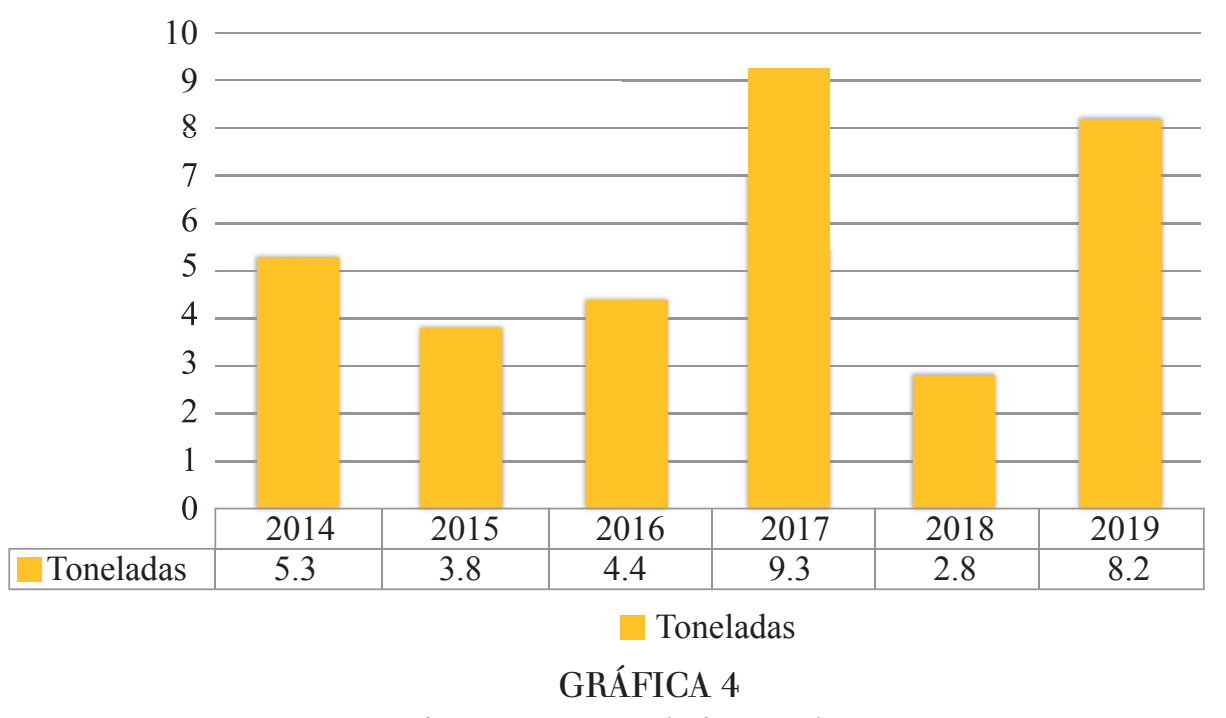

Producción en toneladas por hectárea

Fuente: elaboración propia. 


\section{Prospectiva}

Entre las principales actividades que se han propuesto abordar en este trabajo práctico, y que corresponde a un estudio de caso, es la implementación de nociones particulares sobre desarrollo sustentable, que en muchas ocasiones los campesinos ya aplican aun sin tener el conocimiento científico del área. Un hecho muy particular es la idea de forestar con árboles frutales una porción del área cultivable para hacerla convivir con la producción de maíz, lo cual cubriría, por una parte, la sustentabilidad y, por otra, la opción de nuevos mercados con la producción de frutas. El riego de los árboles se realiza por goteo con la instalación de mangueras y una bomba eléctrica; al respecto, hay dos estanques de agua de lluvia que se encuentran dentro del predio.

Es importante considerar la unión de productores, mecanismo que ya existe en otros lados del país, en los Estados Unidos y en países que conforman la Unión Europea, en donde se permite la colaboración entre competidores bajo la figura de cooperativas y asociaciones sin que se incurra en violaciones a las leyes de competencia. Esto permite reducir costos de aprovisionamiento de insumos, eliminar algunos eslabones en la intermediación, propiciar el cambio en actividades de procesamiento y equilibrar las relaciones en la negociación con distribuidores y grandes consumidores. Todo ello otorga posibilidades de mejora en los ingresos de los productores agropecuarios.

Otros aspectos importantes que surgen como respuesta a las necesidades que plantean los agricultores entrevistados es la adopción paulatina de agricultura por contrato que permitiría minimizar el riesgo en el precio de la cosecha, así como considerar la posibilidad de la compra de seguros de la cosecha contra siniestros.

TABLA 1

Fertilizantes y formas de aplicación

\begin{tabular}{|c|c|c|c|c|c|}
\hline Concepto & $\begin{array}{l}\text { Ingrediente } \\
\text { activo }\end{array}$ & Presentación & $\begin{array}{l}\text { Cantidad } \\
\text { utilizada }\end{array}$ & Costo unitario & Observaciones \\
\hline Semilla & Híbrida mejorada & $\begin{array}{l}60000 \\
\text { semillas }\end{array}$ & 15 & $\$ 2850.00$ & $\$ 4275$, semilla sin tratamiento \\
\hline Insecticida granulado & Imidacloprid $2 \%$ & $10 \mathrm{~kg}$ & 1 & $\$ 550.00$ & Al momento de la siembra \\
\hline Fertilizante de fondo & $\begin{array}{l}\mathrm{N}, \mathrm{P}, \mathrm{K},+ \\
\text { elementos menores }\end{array}$ & $1 \mathrm{~kg}$ & 300 & $\$ 8.40$ & $\begin{array}{l}\$ 2520, \text { al momento de la siem- } \\
\text { bra }\end{array}$ \\
\hline Sellador & Acetoclor $75.3 \%$ & 11 & 4 & $\$ 220.00$ & $\begin{array}{l}\text { \$880, con atrazina, aplicar } \\
\text { después de siembra }\end{array}$ \\
\hline Sellador & Atrazina $90 \%$ & $1 \mathrm{~kg}$ & 2 & $\$ 150.00$ & $\begin{array}{l}\$ 300 \text {, mezclarlo con acetoclor y } \\
\text { aplicar después de la siembra }\end{array}$ \\
\hline Desecante & Glifosato & 11 & 3 & $\$ 80.00$ & $\begin{array}{l}\text { \$240, con sellador en presencia } \\
\text { de maleza emergida }\end{array}$ \\
\hline $\begin{array}{l}\text { Enraizador y co- } \\
\text { sechador de nitrógeno }\end{array}$ & $\begin{array}{l}\text { Azospirillum, } \\
\text { bacillus subtlis }\end{array}$ & 2.51 & 1 & $\$ 285.00$ & $\begin{array}{l}\text { Aplicar una vez nacido el maíz y } \\
\text { que no se vaya a resembrar }\end{array}$ \\
\hline $\begin{array}{l}\text { Control de plagas a } \\
\text { la pata }\end{array}$ & Clorpirifos $480 \mathrm{gr} / 1$ & 11 & 1 & $\$ 170.00$ & $\begin{array}{l}\$ 170 \text {, aplicar mezclado con } \\
\text { enraizador y cosechador de } \\
\text { nitrógeno }\end{array}$ \\
\hline $\begin{array}{l}\text { Primera aplicación de } \\
\text { foliar }\end{array}$ & $\mathrm{N}, \mathrm{Zn}$ & 11 & 0.5 & $\$ 250.00$ & $\begin{array}{l}\$ 125 \text {, aplicar cuando el maíz } \\
\text { tenga de cuatro a seis hojas } \\
\text { verdaderas }\end{array}$ \\
\hline Control cogollero & Permetrina 340 & 11 & 0.5 & $\$ 600.00$ & $\begin{array}{l}\$ 300, \text { mezclar con nitrato de } \\
\text { zinc }\end{array}$ \\
\hline $\begin{array}{l}\text { Primera aplicación de } \\
\text { nitrógeno al suelo }\end{array}$ & Nitrógeno & $1 \mathrm{~kg}$ & 330 & $\$ 7.40$ & $\begin{array}{l}\$ 2442 \text {, aplicar cuando el maíz } \\
\text { tenga seis hojas }\end{array}$ \\
\hline $\begin{array}{l}\text { Recarga insecticida al } \\
\text { suelo }\end{array}$ & $\begin{array}{l}\text { Clorpirifos } 5 \% \\
\text { granulado }\end{array}$ & $20 \mathrm{~kg}$ & 0.5 & $\$ 330.00$ & $\begin{array}{l}\$ 165 \text {, aplicar a las seis hojas } \\
\text { mezclado con nitrógeno }\end{array}$ \\
\hline $\begin{array}{l}\text { Segunda aplicación } \\
\text { foliar }\end{array}$ & Boro & 11 & 0.5 & $\$ 250.00$ & $\$ 125$, aplicar a las nueve hojas \\
\hline Insecticida cogollero & Permetrina 340 & 11 & 0.5 & $\$ 500.00$ & $\begin{array}{l}\$ 250 \text {, aplicar mezclado con boro } \\
15 \%\end{array}$ \\
\hline
\end{tabular}


TABLA 1

Fertilizantes y formas de aplicación (continúa)

\begin{tabular}{|c|c|c|c|c|c|}
\hline Concepto & $\begin{array}{l}\text { Ingrediente } \\
\text { activo }\end{array}$ & Presentación & $\begin{array}{l}\text { Cantidad } \\
\text { utilizada }\end{array}$ & Costo unitario & Observaciones \\
\hline $\begin{array}{l}\text { Nitrógeno, segunda } \\
\text { aplicación al suelo }\end{array}$ & Nitrógeno & $1 \mathrm{~kg}$ & 120 & $\$ 7.40$ & $\$ 888$, aplicar al suelo \\
\hline $\begin{array}{l}\text { Tercera aplicación } \\
\text { foliar }\end{array}$ & $\begin{array}{l}\text { N, P, K hormonas } \\
\text { y E. M. quelatados }\end{array}$ & 2.51 & 1 & $\$ 230.00$ & $\$ 230$, aplicar en hoja bandera \\
\hline $\begin{array}{l}\text { Control de gusano } \\
\text { elotero }\end{array}$ & $\begin{array}{l}\text { Lambda cyalotrina } \\
7.54 \%\end{array}$ & $250 \mathrm{ml}$ & 1 & $\$ 100.00$ & Aplicar mezclado con PQT \\
\hline $\begin{array}{l}\text { Control gusano } \\
\text { picudo }\end{array}$ & Acefate $97 \%$ & $200 \mathrm{gr}$ & 1 & $\$ 100.00$ & $\$ 100$, aplicar mezclado con PQT \\
\hline Control de roya & Tebucozole $23 \%$ & 11 & 0.5 & $\$ 600.00$ & $\$ 300$, mezclar con PQT \\
\hline $\begin{array}{l}\text { Control de hoja ancha } \\
\text { en posemergencia }\end{array}$ & Atrazina $90 \%$ & $1 \mathrm{Kg}$ & 1 & $\$ 150.00$ & $\begin{array}{l}\text { \$150, aplicación al surco para } \\
\text { sellar hoja ancha de todo tipo }\end{array}$ \\
\hline $\begin{array}{l}\text { Control de hoja ancha } \\
\text { en posemergencia }\end{array}$ & Dicamba 480 & 11 & 0.5 & $\$ 300.00$ & $\begin{array}{l}\$ 150 \text {, aplicación al surco para } \\
\text { matar hoja ancha de todo tipo }\end{array}$ \\
\hline & & & \multicolumn{2}{|r|}{ TOTAL } & $\$ 14545.00$ (aprox.) \\
\hline
\end{tabular}

Fuente: empresa distribuidora y productora de agroinsumos "El tractor azul” en el municipio de Tlajomulco de Zúñiga.

\section{REFERENCIAS}

Aguilar, A. y Cabral, A. (2000). Resúmen histórico de la administración agropecuaria en México. Revista Mexicana de Agronegocios, Sociedad Mexicana de Administración Agropecuaria A.C.

Aguilar, J., Illsley, C. y Marielle, C. (2003). Los sistemas agrícolas de maíz y sus procesos técnicos, en G. Esteva y C. Marielle (eds.), Sin maiz no hay pais (pp. 83-122). México: Conaculta.

Castañeda, Z., González, M., Chauvet, S. y Ávila,C. (2014). Industria semillera de maiz en Jalisco. Actores sociales en conflicto. Universidad Autónoma Metropolitana Azcapotzalco.

Cordero, M. C. (2012). Historias de vida: una metodología de investigación cualitativa. Revista Griot, 5(1), $50-67$

Espinoza, F. (2011). Análisis contable de la rentabilidad empresarial. Madrid: Centro de Estudios Financieros.

González, O. y Sánchez, G. (s/f). El desarrollo sustentable y las tendencias en la evaluación de proyectos.

González, A. y Alferes, M. (2010). Competitividad y ventajas comparativas de la producción de Maíz en México. Revista de Ciencias Agrícolas, 1, 3-10.

Grant, R. M. (1997). Dirección estratégica: conceptos, técnicas y aplicaciones. Madrid: S. L. Civitas Ediciones.

Hernández-Sampieri, R. (2015). Metodológia de la investigación. McGraw-Hill.

Huerta, P. (2015). Consideraciones sobre producción y comercio del maíz. Periodismo público y divulgación del conocimiento en Altos Sur de Jalisco. Jalisco: Consejo de Cronistas de la ciudad de Tepatitlán de Morelos.

INEGI-Sagarpa. (2014). Encuesta Nacional Agropecuaria (ENA) 2014. Conociendo el campo. Resultados. México: INEGI-Sagarpa.

Leiva, F. R. (1998). Sostenibilidad de sistemas agrícolas. Agronomía Colombiana, 2-3(15), 181-193.

López, J. (2013). La tecnología campesina en la producción de maiz y el traspatio como estrategia de desarrollo agricola en San Nicolás de los Ranchos, Puebla. Puebla: Colegio de Postgraduados-Institución de Enseñanza e Investigación en Ciencias Agrícolas. 
Longenecker, J., Petty, J., Palich, L., y Hoy, F. (2012). Administración de pequeñas empresas: lanzamiento y crecimiento de iniciativas de emprendimiento. Cengage Learning Editores.

Luna, B., Altamirano, J. y Santoyo, V. (2016). Factores e innovaciones para la adopción de semilla de maíz en Oaxaca. Revista Mexicana de Ciencias Agrícolas, 2995-3007.

Luna, B., Rodríguez-Hinojosa, M. A., Ayala Garay, O. J., Castillo-González, F. y Mejía Contreras, J. A. (2012). Perspectivas de desarrollo de la industria semillera de maíz México. Revista Fitotecnia Mexicana, 35(1).

Núñez, F. y Sempere, J. (2016). Estudio del mercado de producción, procesamiento, distribución y comercialización de la cadena de maíz-harina/nixtmal-tortilla en México. El Colegio de México.

Núñez, L. (2016, diciembre 1). Producción de maíz en México y el mundo. El Economista.

Sagarpa. (2012). Censo. México: Instituto Nacional de Estadística y Geografía.

Sagarpa. (2017). Planeación Agrícola 2017-2030. México: Instituto Nacional de Estadística y Geografía

\section{BY-NC-ND}

